九州大学学術情報リポジトリ

Kyushu University Institutional Repository

\title{
A 3D measurement procedure for internal local crack driving forces via synchrotron X-ray microtomography
}

Toda, Hiroyuki

Department of Production Systems Engineering, Toyohashi University of Technology

Sinclair, I.

Materials Research Group, School of Engineering Sciences, University of Southampton, Highfield, Southampton

Buffière, J.Y.

GEMPPM UMR CNRS

Maire, E.

GEMPPM UMR CNRS

他

http://hdl. handle. net/2324/1807825

出版情報 : Acta Materialia. 52 (5)，pp.1305-1317，2004-03-08. Elsevier バージョン：

権利関係 : 


\title{
A 3D measurement procedure for internal local crack driving forces via synchrotron X-ray microtomography
}

\author{
H. Toda ${ }^{a}$, I. Sinclair ${ }^{\text {b }}$, J.-Y. Buffière ${ }^{\mathrm{c}}$, E. Maire ${ }^{\mathrm{c}}$, K. H. Khor ${ }^{\mathrm{b}}$, P. Gregson ${ }^{\mathrm{b}}$ and T. \\ Kobayashi $^{\text {a }}$ \\ ${ }^{a}$ Department of Production Systems Engineering, Toyohashi University of Technology, Toyohashi, AICHI 441- \\ 8580, Japan (e-mail: toda@ tutpse.tut.ac.jp, tel: +81-532446697, fax: +81-532446690(H. Toda) \\ ${ }^{\mathrm{b}}$ Materials Research Group, School of Engineering Sciences, University of Southampton, Highfield, Southampton \\ SO17 1BJ, UK
}

${ }^{\mathrm{c}}$ GEMPPM UMR CNRS 5510, INSA, Lyon, 20, Av. A Einstein, 69621 Villeurbanne, Cedex, France

\begin{abstract}
Synchrotron X-ray microtomography has been utilized for the in-situ observation of fatigue crack opening/closure during load cycles. High-resolution phase contrast imaging technique has enabled the reconstruction of clear crack images together with the details of microstructural features. Physical displacements of micro-pores in a crack-tip stress field are used to obtain local mixed-mode crack driving forces along a crack front, and its feasibility is confirmed. Complicated crack closure behaviour are observed due to the combined effects of local modes II and III displacements. The technique used provides a highly effective way of assessing local crack driving forces together with supplementary ways of verifying and interpreting it by visualising and quantifying various forms of crack-tip shielding behaviour. The proposed technique is clearly advantageous compared to the limited procedures available in the current literature, where detailed internal information can only be achieved for limited types of material.
\end{abstract}

Keywords: Fatigue; Aluminium Alloys; Image Analysis; Local Crack Driving Force; Synchrotron Radiation 


\section{Introduction and Background}

It is widely accepted that crack closure is a predominant factor determining fatigue crack growth rates via shielding the transmission of external load variations to the crack-tip region [1]. Traditionally, various mechanisms of closure, such as plasticity induced crack closure (PICC) [1,2] and roughness induced crack closure (RICC) [3,4,5], have been discussed on the basis of simplified two-dimensional interpretations of the phenomena, due mainly to their analytical simplicity and the available experimental evidence. In terms of RICC in particular, a basic geometrical modeling approach for simple, regularly deflected crack paths has been proposed by Suresh [3] for example, whilst more complex numerical methods have more recently been considered by Llorca [4] and Parry et al. [6,7]. They have investigated the effects of asperity angle, the absolute scale of asperities and mode III load mixity have been investigated. Whilst valuable micromechanistic insight may be gained from such twodimensional investigations, the true three-dimensional nature of real fracture surfaces cannot be accurately represented.

The present authors have recently examined crack closure process via synchrotron X-ray micro-computed tomography (XMCT) [8], providing an order of magnitude improvement in resolution over previous studies [9]. The work has revealed significant contributions of mode III (i.e. out-of-plane shear) to displacements in the crack wake, as well as local variations in their sense and extent. Since such three-dimensional crack behaviour is difficult to assess via traditional experimental methods (such as post-mortem fractography, or in-situ surface observations), it would appear that in-situ X-ray tomography provides a unique potential to assess real crack closure processes.

The authors' previous XMCT results have shown that relatively large areas of fracture surface contact may particularly arise along ridges and valleys running back from the crack-tip, whilst small, distributed points of contact occur close to the crack-tip [8]. The observed progressive increase in crack surface contact area during unloading suggest that contributions to closure from the various types of contact point may differ significantly not only in extent but also in associated contributions to crack shielding levels. In terms of conventional macroscopic closure measurements based on clip gauges [10] or back-face strain gauges [1], it is assumed that initial deviations of specimen compliance from linearity may be used to define a single opening load. The XMCT results clearly call into question the validity of such simple interpretations of compliance results. Whilst such issues with compliance-based closure measurements have recently been discussed within the literature [11], links 
between specific micromechanical processes and compliance-based measurements remain difficult to interpret.

With XMCT resolutions of the order of one micrometer $[8,12]$ it is possible to utilise microstructural features, such as intermetallic constituent particles and micro-pores in commercial Al-alloys, as gauge marks for quantitative 3D displacement or strain mapping within samples [8]. As such, several valuable features become available for crack opening analysis, such as independent mapping of local mode I, II and III crack flank displacements. Encouraging accuracies have been demonstrated in crack opening displacement (COD) measurements bringing a wide range of potential applications of the X-ray microtomography in fracture studies, particularly in the quantification of failure processes at a microstructural scale.

Numerous experimental reports within the literature have been devoted to measuring crack driving forces under monotonic or cyclic loading. The techniques can be broadly classified into:

(a) Optical methods, such as photoelasticity [13], moiré interferometry [14] and its variations [15,16], speckle photography [17], caustics [18], Raman microprobe spectroscopy [19] and infrared thermal stress analysis [20].

(b) Surface-based microscopy, such as scanning probe microscopy [21] and in-situ stereoimaging in scanning electron microscopes [22].

Furthermore, direct strain measurement from embedded optical fibre strain sensors has recently been reported [23]. Some of the above techniques yield information that is essentially averaged across the through-thickness direction of a specimen $[13,18]$, whilst many of the others reflect surface-specific two-dimensional deformation behaviour. Several techniques enable the assessment of local crack driving forces within a material bulk, specifically, the embedded-multiple-grid moiré method [16], frozen stress photoelasticity [24], Isodyne technique [25], scattered light speckle photography technique [26] and optical fibre strain sensing [23]. Significant limitations exist however, with the embedded-multiple-grid moiré method only providing interior strains at several points with low sensitivity, whilst frozen stress photoelasticity, the isodyne and scattered light speckle photography techniques are limited to transparent materials and/or birefringent materials. Optical fibre strain sensing facilitates measurements between relatively widely spaced points (separations of the order of several millimetres at least) limiting the examination of effects of microstructural features and local crack geometries. A further general drawback of methods which involve embedded particles or fibres is of course the potential perturbation internal stress fields of interest by incorporation of such second phases into a given material

In terms of crack shielding processes, it is well known that when a crack-tip is deflected and/or twisted from its nominal mode I growth direction, effective driving force for growth is typically smaller than that of a 
horizontal planar crack of the same projected area subjected to the same far-field loading. Therefore, a deflected/twisted crack may propagate at an apparently slower rate than the corresponding horizontal planar crack due to the combined effects of crack closure and crack deflection/twisting. Additional crack-tip shielding mechanisms exist, such as microcracking, crack bifurcation, and load transfer interactions with secondary phases. Such phenomena may further reduce the growth rate of a crack. Since the COD and crack contact area measurements of the previous XMCT study of the authors [8] do not provide a direct estimate of the efficacy of shielding the crack tip from applied loads, applying an analogous procedure to the assessment of local crack-tip driving forces distribution would be clearly valuable. A variety of measurements of local physical parameters which have been demonstrated in the previous study [8] may then enable the separation of different closure and non-closure-based contributions to crack shielding. In the present study a commercial high strength aluminium alloy has particularly been investigated, employing three-dimensional reconstructed volume data of internal features for high-density mapping of crack driving forces along a real fatigue crack front. Via detailed examination of three-dimensional characteristics of the crack-tip region (such as the distribution of fracture surface contacts, crack-tip geometry and microstructural features), modes I, II and III crack driving force distributions along the crack front are calculated and discussed in terms of the contribution of near-tip contact to measured driving force distributions.

\section{Experimental and Measurement Methods}

Analyses performed in the present study were closely based on data reported previously [8, 27, 28]; as such only major points are summarised here.

\subsection{Sample preparation}

To perform high resolution microtomographic imaging at the submicrometer level, the test sample cross section was required to be smaller than $1 \times 1 \mathrm{~mm}^{2}$. In order to obtain meaningful information from such a small sample, conventional single-edge notched $(\mathrm{SEN})$ specimens of $5 \times 20 \times 130 \mathrm{~mm}$ with a fatigue pre-crack were prepared in accordance with ASTM E647-95A [29], with a small parallelepiped specimen (typically $0.8 \times 0.9 \times 25 \mathrm{~mm}$ ) then being carefully extracted at the crack-tip of the original SEN specimen to produce a steady state fatigue crack for tomographic imaging. The original specimens were taken from a rolled plate of a 2024-T351 aluminium alloy, 
tested in the L-T orientation. The final applied load was $6 \mathrm{MPa} \sqrt{\mathrm{m}}$. A single overload of $66 \%$ was applied at the last stage of precracking.

The value of the ratio $a / W$ ( $a$ : crack length, $W$ : width) of the small parallelepiped specimen was 0.68 on average. Note that since the original plastic zone size was about $W / 10$ and $W / 20$ for the maximum and intermediate loading steps, respectively, the plane strain condition should still predominate extensively for subsequent tomography observations.

\subsection{Tomographic imaging}

High resolution X-ray tomography was performed at the X-ray imaging and high-resolution diffraction beamline, ID-19, of the European Synchrotron Radiation Facility (ESRF) in Grenoble, France. A material test rig was set approximately $145 \mathrm{~m}$ from the X-ray source of size $100 \times 25 \mu \mathrm{m}$ in size. A monochromatic X-ray beam having a photon energy of $20 \mathrm{keV}$ from a vertical multilayer monochromator was used for the experiments, giving an incident/transmitted intensity ratio of at least $10 \%$. A cooled $2048 \times 2048$ element CCD detector was positioned $50 \mathrm{~mm}$ behind the sample, thereby setting the imaging system sensitive to phase modulation in addition to classical absorption contrast [27]. In total 1,500 radiographs, scanning 180 degrees, were taken around the loading axis at 0.12 degree increments for each load level. The whole cross section of the specimens, and a height of $210-280 \mu \mathrm{m}$ containing the crack were entirely captured on the CCD camera. The in-situ loading stage allowed specimens to be scanned under load, with a series of results being obtained for loading and unloading each sample to $K_{\max } \sim 4.69 \mathrm{MPa} \sqrt{\mathrm{m}}$ in $20 \%$ increments.

\section{Image data handling}

\subsection{Reconstruction}

Image slices were reconstructed from the series of projections based on a conventional filtered backprojection algorithm [30] using the HST program developed at the ESRF by Andy Hammersley. Grey values in each dataset were calibrated so that variation between the most opaque and transparent voxels could be expressed within an 8 bit grey scale range. Isotropic voxels (3D picture element equivalent to the $2 \mathrm{D}$ pixel) with an edge size of $0.7 \mu \mathrm{m}$ 
were achieved in the reconstructed slices.

\subsection{Volume rendering}

In order to measure three-dimensional locations of the crack-front and microstructural features in the bulk aluminium, the tomographic dataset was thresholded by a three-dimensional 'seed growth' technique [8] and labelled utilizing a grey value for each 3D feature of interest. In terms of the accuracy of the location measurements, the selection of optimum thresholding condition is a key issue. The phase contrast effect generates distinct light and dark fringes at an interface, which was set to reach almost the entire grey scale range. In such a case, it is interesting to adopt a mean value between the grey values of the neighbouring phases as a threshold value to determine the location of the associated interface. Such a mean contrast value was identified as 144.9 in the grey scale range between 0 (black) and 255 (white) for a crack-tip region of the present dataset in a preliminary investigation [8]. A thresholding tolerance value of 160, which is slightly higher than the mean value, was used in the previous study to make crack volume measurements. The exact mean value was however adopted in this study in order to determine crack-tip locations with the least error. In terms of thresholding and tracking microstructural features and micro-porosity for internal displacement/strain measurement (see following section), mean grey values at the interface of a given micro-pore was found to depend on its diameter, because the inner fringe associated with interface phase contrast overlaps in such small objects (typically $3-11 \mu \mathrm{m}$ in diameter). However it was considered reasonable to apply the same thresholding value to the segmentation of micro-pores due to the weak dependency of the measured centroid locations of spherical microstructural features on thresholding tolerance which was revealed in the preliminary investigation [8].

\subsection{Calculation of local crack driving forces}

In the present work densely distributed microstructural features intrinsic to the commercial Al-alloy microstructure have been utilised for crack driving force measurements within the crack-tip stress field [8]. Whilst high-resolution tomographic observation was utilised, the voxel size itself may seem to be an important issue when compared to crack-tip opening displacement (CTOD) levels in such a material. However, it has been shown that high contrast features occupying areas as small as one-tenth of a voxel size can be identified by the use of phase modulation [27] in addition to classical absorption contrast. By analogy with the previous report, substantial detectability to a scale of $100 \mathrm{~nm}$ or less may be expected for the isotropic voxel with a $0.7 \mu \mathrm{m}$ edge used in this 
case. This value is considered reasonable to determine the location of a sharp crack-tip fairly accurately. Previous work has shown that centroid spacings of approximately spherical microstructural features may lead to reliable and robust COD measurements [8], with reproducibility in object separations being achieved to a tenth of the voxel size, independent of image thresholding conditions.

\subsubsection{Centre of gravity measurement of micro-pores}

As reported in the previous paper [8], owing to the high resolution X-ray detector system, the fine details of a fatigue crack and surrounding microstructural features of the aluminium, such as micro-pores and intermetallic compound particles, could be clearly visualised in the current tomographic dataset (Detail levels are reasonably similar to a high magnification optical microscope image. See for example [12] for a direct comparison on an aluminium alloy). Micro-pores distributed within a crack-tip stress field are sampled, with the centre of gravity of each micro-pore then being utilized as a displacement gauge marker to estimate local crack driving force. $x, y$ and $z$ coordinates of each micro-pore were calculated with the right edge (as shown later in Fig.4) of the crack front line as its origin. Since the shape of the micro-pores is usually spherical, the partial volume effects along the surface of each pore have little effect on the determination of the gravity centre of the pore.

The number of micro-pores located in the volume sampled was $\sim 800$. The resultant average interval of measuring points is $0.78 \mu \mathrm{m}$ in the direction of the crack front, thereby offering a high-density crack driving force mapping which may enable the assessment of local shielding phenomena such as crack deflection/twisting, crack closure and particle/crack interaction. The centre of gravity measurement was performed automatically using dedicated software developed by Dr. Luc Salvo at the GPM2 Laboratory INP, Grenoble.

\subsubsection{Determination of crack front location}

In the present study, the crack-tip location was assumed to be the centre of gravity of each crack-tip voxel. When there were more than two crack-tip voxels stacked in the loading direction, the midpoint of two centres of gravity of the lowest and the uppermost voxels was assumed to be the crack-tip location. Crack-tip location at an arbitrary point between two neighbouring crack-tip locations along the crack front line was then calculated by three-dimensional linear interpolation.

If voxel size is infinitesimally small and only absorption contrast is reflected in measured voxel values, adopting a threshold value which is close to the linear attenuation coefficient of the matrix aluminium may enable the reasonable determination of crack front location from the tomography data. However, the voxel size achieved 
by the present tomography setup is as large as $30 \%$ of the average COD at the crack-tip. In addition, the imaging conditions of the present study inherently includes light and dark fringes at each crack edge due to the phase contrast effects between air and aluminium, significantly complicating the determination of crack-tip location. Associated location measurement errors can exceed half the voxel size at maximum (i.e. $0.35 \mu \mathrm{m}$ ) in all the three directions. Also, the so-called partial volume effect [31] may be crucial, which brings a transition area with a grey value ramp over several voxels in width at an interface between radiographically dissimilar phases. Provided that the tolerance value applied is appropriate, the partial volume effect then can also cause a maximum error of half the voxel size in the determination of the crack-tip location. The average micro-pore/crack-tip separation distance in the crack-tip volume sampled in this work was found to be $82 \mu \mathrm{m}$, which is more than 230 times larger than these errors. As such, the average errors due to such uncertainty in location accuracy of the crack-tip (i.e. a shift of a crack-tip location by $0.35 \mu \mathrm{m}$ ) were small in a final crack driving force result; corresponding to $\sim 0.23,0.094$ and $0.25 \%$ in the calculated mode I, II and III stress intensity components respectively, i.e. reasonably negligible.

\subsubsection{Calculation of crack driving force distributions along the crack front}

As crack tip driving forces were to be extracted from the elastic $K$-dominated field of the cracks of interest, it is necessary to avoid errors associated with the plastic deformation of the matrix, the transition between the $K$ dominant and $J$-dominant regions and the transition between $K$-dominant and remote boundary conditiondominated regions. As such, only micro-pores within the nominal plane strain $K$-dominated elastic stress field were sampled for the analyses. According to the three-dimensional modified boundary layer analysis performed by Kim at al. [32], plane strain constraint should remain almost constant along the crack line except for the regions which are within 5\% of the thickness from each specimen surface. Such sub-surface regions were excluded from the measurement in this study. In terms of locating the $K$-dominated distance from the crack tip, the well-known mode I plastic zone radius equation [33] for plane strain was firstly used to estimate the size of the crack-tip yielding zone, $\gamma_{p}$. Yield strength value of $325 \mathrm{MPa}$ was used for the calculation. The farthest point of the plastic zone boundary is located at $\theta=87$ degrees from the initial crack plane, which yields a $\gamma_{p}$ value of $53.6 \mu \mathrm{m}$ at this angle.

In general, the crack-tip elastic stress field outside the plastic zone can be expanded as a series in half integer powers of the distance from a crack-tip, $r$. Higher order terms vary from one specimen geometry to another, and one loading mode to another. In terms of an edge crack problem in a finite plate subjected remotely to a uniform tensile stress, which is close to the situation of this study, Xiao and Karihaloo [34] first solved the problem 
recently by superposing the analytical solution for a semi-infinite crack, and a numerical solution with free crack faces. The general solution for the displacement resolved perpendicular to the loading direction is expressed as a Williams expansion of the following form:

$$
u_{z}=\sum_{n=1}^{\infty} \frac{r^{n / 2}}{2 \mu} a_{n}\left[\left(\kappa+\frac{n}{2}+(-1)^{n}\right) \cos \frac{n}{2} \theta-\frac{n}{2} \cos \left(\frac{n}{2}-2\right) \theta\right]
$$

where $\mu$ is shear modulus and $\kappa=3-4 v$ for plane strain. The coefficients, $a_{n}$, are determined to meet the boundary conditions using weighted residual boundary techniques. In the direction of the initial crack line (i.e. $\theta=0$ ), and at a distance of $r=82 \mu \mathrm{m}$ from the crack tip (which is the average distance between micro-pores sampled and the crack-tip , as shown later in Fig. 4) the contributions from $a_{2}, a_{3}, a_{4}$ and $a_{5}$ were calculated to be $5.1 \times 10^{-3}, 6.3 \times 10^{-6}$, $1.3 \times 10^{-7}$ and $3.5 \times 10^{-11}$, respectively. It can be concluded that the displacement contributions from the terms higher than the $r^{3 / 2}$ term are negligible for the displacement measurements of this study. Hartranft and Sih provided the general solution of three-dimensional crack problems using eigenfunction expansions [35]. They showed that the near-tip field in a three-dimensional field possesses the same functional relationships as the in-plane problem in terms of $r$ and $\theta$. In the present study therefore the three-dimensional displacement components [32] reduce to plane relationships:

$$
\begin{gathered}
u_{y}=\frac{1+v}{E} \sqrt{\frac{2 r}{\pi}}\left(k_{I} h_{11}+k_{I I} h_{12}\right)+\frac{T r}{E} h_{13} \\
u_{z}=\frac{1+v}{E} \sqrt{\frac{2 r}{\pi}}\left(k_{I} h_{21}+k_{I I} h_{22}\right)-\frac{T r}{E} h_{23} \\
u_{x}=\frac{1}{E} \sqrt{\frac{2 r}{\pi}} k_{I I I} h_{31}
\end{gathered}
$$

where

$$
\begin{gathered}
h_{11}=\cos \frac{\theta}{2}\left\{1-2 v+\sin ^{2}\left(\frac{\theta}{2}\right)\right\}, \quad h_{12}=\sin \frac{\theta}{2}\left\{2(1-v)+\cos ^{2}\left(\frac{\theta}{2}\right)\right\}, \quad h_{13}=\left(1-v^{2}\right) \cos \theta \\
h_{21}=\sin \frac{\theta}{2}\left\{2(1-v)-\cos ^{2}\left(\frac{\theta}{2}\right)\right\}, \quad h_{22}=\cos \frac{\theta}{2}\left\{1-2 v-\sin ^{2}\left(\frac{\theta}{2}\right)\right\}, \quad h_{23}=v(1+v) \cos \theta, \\
h_{31}=2(1+v) \sin \frac{\theta}{2}
\end{gathered}
$$

where $E$ is Young's modulus, $T$ is $T$ stress and $k_{I}, k_{I I}$ and $k_{I I I}$ are local stress intensity factors in modes I, II and III. 
Therefore $k_{I}, k_{I I}$ and $k_{I I I}$ are inversely obtained from the following equations:

$$
\begin{gathered}
k_{I}=\frac{1}{1+v} \sqrt{\frac{\pi}{2 r}}\left(\frac{E h_{22} u_{y}-E h_{12} u_{z}-T r h_{41}}{h_{42}}\right) \\
k_{I I}=\frac{1}{1+v} \sqrt{\frac{\pi}{2 r}}\left(\frac{E h_{21} u_{y}-E h_{11} u_{z}-T r h_{51}}{h_{52}}\right) \\
k_{I I I}=E \sqrt{\frac{\pi}{2 r}} \frac{u_{x}}{h_{31}}
\end{gathered}
$$

where

$$
h_{41}=h_{13} h_{22}+h_{12} h_{23}, h_{42}=-h_{52}=h_{11} h_{22}-h_{12} h_{21}, h_{51}=h_{13} h_{21}+h_{23} h_{11}
$$

To obtain crack driving force data from a crack, two sets of micro-pore location data were chosen, corresponding to the minimum load and another higher load level. Micro-pore data sets comprised coordinates, volumes and surface areas of the corresponding micro-pores. Coordinates were then superposed to calculate displacements of each micro-pore between the two load levels. The crack driving forces at the nearest crack-tip, $k_{I}$, $k_{I I}$ and $k_{I I I}$, were inversely estimated for each micro-pore using equations (3)-(5). In this case, the precise superposition of two sets of micro-pore location data is crucial to calculate the crack driving forces exactly. An error incidental to the finite voxel size can however be of the order of the voxel size (i.e. $0.7 \mu \mathrm{m}$ in this study) in all three directions. The average absolute displacement of all the micro-pores in the crack-tip sample volume was approximately $0.50 \mu \mathrm{m}$ in the mode I direction between the minimum and maximum external loads, implying that such errors in superposition must be addressed for the crack driving force calculation. Also, rotation of the specimen due to slight alignment shifts in the tensile rig during loading and unloading may cause some difficulty in matching the two volume sets.

Figure 1 schematically illustrates the effects of mismatching between two volume sets. For example, if a volume set captured at a higher load level is shifted by the error in superposition by distance, $d_{z}^{e}$, in the $z$ direction (i.e. rigid body translation), the error in a final mode I crack driving force result, $\Delta k_{z}{ }^{e}$, is positive for the upper quadrant in Fig.1, while that for the lower quadrant is negative. The crack driving force data points are therefore bipolarised. Fig.2 is a schematic example of such bipolarisation in the case of rotational mismatching, where a volume set is intentionally shifted in the $y$ direction by rotating one volume relatively against another volume around the $z$ axis for demonstration purposes. Since a large number of micro-pores are relatively uniformly distributed in the crack-tip volume sampled, the numbers of the micro-pores located in the upper and lower 
quadrants may be identified as essentially equal and the average effects of the error in registration can be assumed to be nearly identical between them. Linear equations in the form of $c_{i}(x)=\mathrm{a}_{i} x+\mathrm{b}_{i}\left(i: x, y\right.$ or $z, \mathrm{a}_{i}$ and $\mathrm{b}_{i}$ : constants and $x$ : distance in the $x$ direction) were used as correction functions to eliminate the errors in superposition, whereby the relative shifts in $x, y$ and $z$ translational directions and relative rotations around the three axes are corrected simultaneously. Three pairs of the constants, $\mathrm{a}_{i}$ and $\mathrm{b}_{i}$, were determined by the simplex method so that the variances in mode I, II and III crack driving force data in each mode were minimized.

Figure 3 shows an example of apparent variation in $k_{I}$ calculated by the above sequence as a function of azimuth angle of marker points (i.e. the micro-pores). It is clear that an unrealistic dependency on azimuth angle exists: given the low/near-threshold loading conditions of the test, it is likely that plastic deformation will in fact be rather localised, with slip band activity in a specific direction(s) extending to distances greater than the nominal plastic zone size [36]. In relation to Figure 3 it is apparent that there are distinct non-linear deformation effects at angles of about -19 and +58 degrees to the crack-tip direction, consistent with intense slip bands extending beyond the predicted plastic zone in these directions. To avoid the effects of such localized plastic deformation, the direction range where the angular dependency was observed (i.e. $-71 \sim+111$ degrees ahead of the crack-tip) was excluded from the analyses.

\subsection{Assessment of crack opening profile}

The volume of the open crack was extracted from the tomographic dataset by thresholding. COD measurements were sought using two-dimensional representations of spatial distribution of opacity which were produced by a direct ray casting technique $[37,38]$. This representation enables a local COD profile on an arbitrary cross section, including a crack-tip region as described in Ref. [8]. Comparison of such representations allows the tendency of crack driving force variations data to be unambiguously identified and interpreted as has been already demonstrated in the preliminary investigation [8].

\section{Qualitative analyses of the tomography data}

\subsection{Micro-pore distribution near the crack-tip}

Figure 4 shows the three-dimensional distribution of micro-pores within part of a rendered volume $(270 \times 270$ 
$\times 910$ voxels) encompassing a crack front line. The image was taken at the minimum load (i.e. $K=0.42 \mathrm{MPa} \sqrt{ } \mathrm{m}$ ).

Large number of dark micro-pores is observed in the volume. Mean porosity was $0.254 \%$ and mean diameter was $7.2 \mu \mathrm{m}$ (expressed as equivalent diameter for sphere of equal volume) for the volume in Fig. 4.

There are two possible formation mechanisms of the micro-pores. One is cavity formation associated with broken brittle intermetallic particles which might have failed during the various shaping processes associated with plate fabrication. Such micro-pores may be expected to exhibit an elongated shape as are observable on enlarged views such as Fig. 5 of the companion paper [8]. The other void formation mechanism may be identified as homogeneous or heterogeneous precipitation of hydrogen at a high temperature after the final shaping process (i.e. during the solution treatment), which may produce relatively spherical micro-pores. Such micro-pores are also easily observable in the microstructure, being dissociated from intermetallic particles (at the $0.7 \mu \mathrm{m}$ resolution at least). Figure 5 shows a two-dimensional projection of all the micro-pores shown in Fig. 4 onto a plane perpendicular to the loading axis. Only the centres of gravity of the micro-pores are shown to avoid confusion. It may be noted that the micro-pores appear to exhibit a spatial distribution pattern, being lined up in approximately straight bands in the growth direction. The measured approximate spacings of the bands, corresponding to $15 \sim 50$ $\mu \mathrm{m}$, shows agreement with a mean grain size of $34 \mu \mathrm{m}$ measured in the $x$ direction (mean linear intercepts). As the tomography specimens were sampled in the L-T orientation as was explained in 2.1, the pancake-like grain structure of the alloy will give a similar distribution pattern when grain boundaries are projected in the L direction during rolling. Although the location of grain boundaries should be identified by other sophisticated 3D measurement methods, such as Gallium wetting techniques [12], it may still be inferred that these lines correspond to grain boundaries of the aluminium. The grain boundaries are in general a high diffusivity path for hydrogen. The formation of such fairly spherical micro-pores along underlying grain boundaries suggests that the major formation mechanism of the micro-pores is not cavity formation among fragmented particles during fabrication but hydrogen precipitation at the grain boundaries.

In Fig. 5, a crack front line was also superimposed in grey by projecting the crack front line onto the same plane as the micro-pores. The crack's extension varied remarkably with $x$ coordinate (i.e. along the crack front line) within the current tomography specimen. In addition to the retarded sections of crack front observed at the left edge and around the centre of the specimen (i.e. $x=300 \mu \mathrm{m}$; labelled 'R1'), localized crack advance projecting sharply (of the order of $10 \sim 35 \mu \mathrm{m}$ in width) ahead of the general crack line with loading can clearly be observed, as indicated by label 'A1' to 'A4' in the figure. An interesting feature to note is distinct correspondence between the localised crack advance (e.g. crack-tip segment 'A2', 'A3' and 'A4') and the spatial distribution of the micro- 
pores. This suggests that local crack advance occurs preferentially in some grains, sometimes leaving unfractured ligaments between those fractured grains. Such crack front geometry variations may be expected to result in local variations in crack driving forces. In order to propagate the overall crack front, the mode I crack driving force near the uncracked ligaments will be enhanced, while that at the locally advanced crack sections will be effectively reduced due to the constraint of surrounding uncracked ligaments. Post-overload crack growth retardation behaviour has been sometimes attributed to such crack front profile variations after overloading [39]. The local crack advance observed in the present study may be identified to be peculiar to the overloaded specimen by comparing to corresponding constant amplitude tests. It has been also reported that the crack front geometry variations were linked to under-ageing conditions of aluminium alloys. Note that this is the case of the present 2024-T351 material [40]. Although the effect of crack front geometry variations on crack growth have been recognised by other authors, quantitative evaluation of this effect has always been difficult to obtain due to the three dimensional nature of the problem and difficulties in separating stress states. Synchrotron X-ray microtomography provides a unique possibility to approach this issue in a quantitative manner.

\subsection{Crack morphology}

Figure 6 shows the crack volume within Fig. 4 taken at $K=0.42 \mathrm{MPa} \sqrt{\mathrm{m}}$ which has been visualized by removing underlying aluminium, intermetallics and micro-pores. Fig.6 (a) is a front view taken from a point on the extension plane of the crack, being rendered using front and side diffuse light sources to highlight the threedimensional character of the crack [41]. Fig. 6 (b) shows a front illuminated rendering of the same crack segment viewed from the loading direction, emphasizing crack closure and uncracked ligaments in the crack wake in white. The macroscopic geometry of the crack's leading edge can be clearly correlated with complex and threedimensional topology of the crack. In the first instance, the three-dimensional observation reveals that multiple crack planes overlapping over more than $80 \mu \mathrm{m}$ in the $x$ direction were involved in the crack growth around the retarded crack segment highlighted at 'R1' in Fig.5. Although it is outside the crack volume shown, the overlapped crack planes are found to gradually converge at a distance of $200 \sim 300 \mu \mathrm{m}$ behind the crack front line via a segment of crack plane which is twisted about the overall crack growth direction. This sort of threedimensional crack bifurcation was frequently observed on close inspection of the tomography data, such as Fig.5 of the previous paper [8], revealing that such complex arrangements of crack planes probably interact intricately with underlying grain structure. The tilting and twisting of the crack plane as it enters a grain has been imaged 
using the Ga decoration technique by Ludwig et al. [12]. In their case, it was also found that such a complex crack morphology corresponded to a retardation on the crack front. This effect has also been evidenced in an Al-Li alloy [42]. Another feature to note is significant local mode II crack deflection (i.e. crack deviation about an axis parallel to the crack front), as particularly shown around the crack-tip segment labelled 'A4' in Fig.5 and several other points.

A set of the same front illuminated renderings as Fig. 6 (b) is shown in Fig.7, illustrating the variation of the area of fracture surface contact during loading the specimen from the minimum to the maximum loads. Comparison of these images allows the two kinds of fracture surface contact to be unambiguously identified as has been already clarified in the preliminary investigation [8]. Areas of contact show evidence of being arranged in the crack growth direction, as observed behind the crack-tip segment labelled 'A4' in Fig.5. It has been already clarified that ridges and valleys of the fracture surface running in the crack growth direction appear to contribute to crack closure due to the presence of local mode III displacement [8]. The groups of such relatively dense and large contact areas in the 'far' crack wake appear quite distinct from small points of contact (typically $\sim 7 \mu \mathrm{m}$ ) near the crack-tip which are dispersed irregularly, corresponding to subtle undulations of the crack plane. Even at the maximum load, many small points of closure still remained near the crack-tip. Since such near tip contact may directly affect local effective stress intensity range at a crack-tip (i.e. as influence on stress intensity of point loads along a crack wake fall off steeply with distance from the crack tip), the small near-tip contacts may still cause significant benefits to fatigue crack growth resistance.

\section{Crack driving force calculations}

Calculated variations of local crack driving forces in modes I, II and III along the $x$ axis (i.e. the direction along the crack front line) are shown in Fig.8. The crack driving forces, especially the mode I data, are seen to vary to some extent with $x$. As described above, the accuracy of the driving force calculation depends on how accurately the registration is performed for the two sets of volume data that are being superposed. The interpretations of the respective data points will be discussed later by assessing the crack driving forces from an alternative approach, i.e. near-tip crack opening profiles by the ray casting technique. It should be noted that significant scatter in the crack driving force measurements is especially seen in the central region and the rightmost edge of the crack front, with $k_{I}$ sometimes showing negative values, which is considered theoretically unrealistic. In such specifics area of the crack, there are in fact pairs of crack planes which overlap. It can be 
inferred that the particular scatter within these areas is directly associated with complex three-dimensional deformation around such bifurcated crack morphologies.

Variations of average local crack driving forces along the crack front line, $\overline{k_{I}}, \overline{k_{I I}}$ and $\overline{k_{I I I}}$, are shown in Fig.9. $\overline{k_{I}}, \overline{k_{I I}}$ and $\overline{k_{I I I}}$ measured at the maximum applied stress intensity of $4.69 \mathrm{MPa} \sqrt{\mathrm{m}}$ (i.e., $K_{I}-K_{\min }=4.27$ $\mathrm{MPa} \sqrt{\mathrm{m}}$ ) are $3.2,8.0 \times 10^{-2}$ and $6.7 \times 10^{-1} \mathrm{MPa} \sqrt{\mathrm{m}}$, respectively. The $\overline{k_{I}}$ value is about $74 \%$ of the externally applied stress intensity factor, implying the apparent contributions from near-tip crack closure shown in Fig. 7 and/or other shielding mechanisms such as crack deflection/twisting. The mode I crack driving force increased monotonically on loading, with the initial increment being slightly smaller than those of the later stage of loading. This is fairly consistent with the previous report [8] where both the average COD measured over the whole crack and the local COD near marker particles showed similar tendencies. In Fig.9 the mode II crack driving force is almost negligible whilst a significant mode III component is generated on initial loading to a $K_{I}$ level of 2.71 $\mathrm{MPa} \sqrt{\mathrm{m}}$ (i.e., $K_{I}-K_{\min }=2.29 \mathrm{MPa} \sqrt{\mathrm{m}}$ ). These tendencies are also consistent with those of the previous report in terms of the variations of average and local COD values in the crack wake and the measured area of fracture surface contact.

Whilst Fig. 9 gives a valuable indication of overall crack driving force and does suggest a distinct attenuation of stress intensity range experienced at the crack tip, it is clear that the crack geometry is complex, with local variations in crack length, angle, contact point distribution etc. contributing to the results for individual points along the crack. It may then be noted that identification of a simple, single crack closure point in the present data (as generally sought in macroscopic closure determinations via specimen compliance) may be irrational. Furthermore, comparison of the attenuation of stress intensity range in the present tomography samples with closure levels that may be determined for conventional large coupons using bulk compliance data should be cautious, as the present samples encompass a short amount of the crack wake and there will have been a loss of elastic constraint by the simple act of cutting the tomography sample from the original test piece. Both factors would be expected to reduce the apparent efficacy of crack shielding due to closure and would require further quantification. It is however valuable to note that an attenuation of crack driving force has been directly observed in material displacements at the crack tip, and micromechanical aspects of this behaviour may be further investigated in at least a semi-quantitative manner from the various micro-pore displacement measurements.

Of the various micro-pores sampled, three micro-pores which exhibit representative tendencies in crack driving force variations are shown in Fig.10. Point A is the most typical point which shows an almost linear increase in $k_{I}$ 
with external loading. Although the generation of local mode III driving force is confirmed in Fig.10 (c), no discernible crack closure occurred at point A. $k_{I} / K_{\max }$ at the maximum load is approximately 0.8 at point A, suggesting that another form of crack-tip shielding phenomenon may be operative at this location. Since shielding appeared to operate uniformly over the whole load level, it may be presumed to be the influence of local crack extension variations and/or crack twisting as the mode II opening component is almost negligible. On the other hand, at points B and C, $k_{I}$ at the maximum stress intensity is close to $K_{\max }$, implying that crack closure may be the key shielding mechanism. Point B shows an apparently 'classical' effect of near-tip crack closure, where the local mode II displacement (i.e. which may then generate asperity offset and hence contact) appears significant. The mode I crack driving force was kept almost constant below $K_{I}-K_{\min }=2.29 \mathrm{MPa} \sqrt{\mathrm{m}}$. On the other hand, a more complex variation in $k_{I}$ is observed at point $\mathrm{C}$ where the change in $k_{I}$ with $K_{I}$ was 'interrupted' at $K_{I}-K_{\min }=3.54$ $\mathrm{MPa} \sqrt{\mathrm{m}}$. The crack closure which gave rise to this shielding may be identified with local mode II displacement as a reverse of mode II sliding occurred at this load level without obvious variation in $k_{I I I}$. This may be due to mode II deflection locally occurring near the crack-tip, which is observed in Fig.6 (a). $k_{I}$ however starts changing again below $K_{I}-K_{\min }=2.29 \mathrm{MPa} \sqrt{\mathrm{m}}$ with a higher apparent rate than that seen at high stress intensities. In spite of the rapid decrease of $k_{I}, k_{I}$ and $k_{I I}$ are still larger than those of point A even at the minimum load. It can be inferred that although initial sticking at some asperity wedge may have been lost to some extent during the load cycle, probably due to the mode III sliding, the fracture surface contact is still effective in reducing $k_{I}$.

These inferences can be confirmed by looking at the variations of crack opening profile on corresponding cross-sections by the ray casting algorithm plotted back from the crack-tip, as shown in Fig. 11. Fig.11 (a) and (b) correspond to points $\mathrm{B}$ and $\mathrm{C}$, respectively. Distinct fracture surface contact occurred at point $\mathrm{B}$ at $K_{I}=2.71$ $\mathrm{MPa} \sqrt{\mathrm{m}}$ (i.e., $K_{I}-K_{\min }=2.29 \mathrm{MPa} \sqrt{\mathrm{m}}$ in Fig.10), as can be seen in Fig. 10 (a). The COD in the vicinity of the crack-tip is almost kept unchanged below $K_{I}=2.71$ due to the distinct fracture surface contact between 23 and 32 $\mu \mathrm{m}$ from the crack-tip which can be also clearly confirmed in the projected crack image of Fig.6 (b). The length of the fracture surface contact is $20-30 \%$ of the distance from the crack-tip, suggesting the area of this extent may be sufficient to sustain the applied load. On the other hand, similar onset of fracture surface contact can be observed at point $\mathrm{C}$ at $K_{I}=3.96 \mathrm{MPa} \sqrt{\mathrm{m}}$, while this fracture surface contact contracted below $K_{I}=2.71 \mathrm{MPa} \sqrt{\mathrm{m}}$, with the COD level ahead of and behind the contact area being correspondingly reduced over the distance range shown in the figure. This decrease in the COD obviously corresponds to the decrease in $k_{I}$ shown in Fig. 10 (a). The corresponding rightmost edge of Fig.7 indicates that the two-dimensional shape of contact area varied between $K_{I}$ $=0.42$ and $1.17 \mathrm{MPa} \sqrt{ }$ m, suggesting that the tendency within this area is different from point to point. Overall it 
may be seen that the crack opening profile analyses and other 3D and 2D observations can confirm findings from the crack driving force data, illustrating the unique capabilities of high-resolution microtomography and the variety of analysis methods offered by such an information-rich experimental approach.

\section{Summary}

High-resolution synchrotron X-ray microtomography has been utilized to estimate local mixed-mode crack driving forces at arbitrary crack-tip locations. To achieve this, in-situ observation of fatigue crack opening and closing behaviour in a high strength aluminium alloy was performed at the European Synchrotron Radiation Facility. The high resolution experimental setup and the utilization of phase contrast imaging conditions have enabled the reconstruction of the distinct 2D slices and 3D renderings of crack, micro-pores and intermetallic particles with excellent image quality (isotropic voxels with a $0.7 \mu \mathrm{m}$ edge). Micro-pores which were densely distributed in the alloy were sampled in a plane strain elastic crack-tip stress field. It's fairly obvious that the pores are linked to the grain structure, but we don't have explicit evidence/micrographs to show it at the moment. Plastic zone and slip bands which extended beyond the plastic zone expected by conventional prediction were excluded from the analysis. Centroid locations for each micro-pore were tracked against the corresponding crack-tip as a function of applied load thereby allowing to estimate the variations of modes I, II and III crack driving forces along the crack front line. As such, high density mapping with a micrometer level resolution has been realized by the procedure. The accurate registration between pairs of reconstructed volumes was a key issue in deriving meaningful values of the crack driving forces. The calculated crack driving force data provided several valuable insights into the understanding of actual three-dimensional crack opening and closing behaviour, which can be drawn neither by post failure analyses, nor by other currently available procedures for crack driving force determination. For example, complex crack closure behaviour was observed at an internal specific crack-tip point due to the combination of modes II and III displacements of mating asperity wedges. This behaviour could be confirmed and interpreted from different aspects of the data; crack opening profiles on corresponding crosssections extracted by the ray casting volume rendering algorithm and mapping of the crack volume. Overall, high resolution in-situ synchrotron X-ray microtomography offers a highly effective way of assessing the local mixedmode crack driving forces. The interpretation of crack driving force variations is also enabled by visualising and quantifying various internal phenomena which contribute to crack shielding behaviour. The method proposed in 
the present study is considered to be a robust procedure being appropriate for automatic high-density mapping in the case of practical materials which inherently contain small and closely interspersed intermetallic particles or micro-pores.

\section{Acknowledgement}

The ID19 staff is acknowledged for assistance during the experiment. One of the authors (H.T.) would like to acknowledge support through the JSPS - Royal Society Bilateral Programme. The support of Pechiney CRV to K.H.K., the use of the location measurement software by Dr. Luc Salvo, and stimulating discussions with J.C Ehrström in Pechiney CRV are also gratefully acknowledged.

\section{References}

[1] Elber W. In: ASTM STP486, Pensylvania: American Society for Testing and Materials, 1971; 230.

[2] Newman JC. In: ASTM STP590, Pensylvania: American Society for Testing and Materials, $1976 ; 281$.

[3] Suresh S. Metallurgical Transactions. 1983; 14A: 2375.

[4] Llorca J. Fatigue \& Fracture of Engineering Materials \& Structures. 1992; 15: 655.

[5] Beevers CJ., Carlson RL. In: Editor Smith RA. Fatigue Crack Growth, 30 Years of Progress, Pergamon Press, $1984 ; 89$.

[6] Parry MR., Syngellakis S. and Sinclair I. In: Editors Brebbia \& Selvadurai. Damage and Fracture Mechanics 2000, WIT Press, 2000; 313.

[7] Parry MR., Syngellakis S., and Sinclair I. Mater. Sci. Eng. A: 2000; 291: 224.

[8] Toda H., Sinclair I., Buffière J-Y., Maire E., Connolley T., Joyce M., Khor KH., Gregson P. Phil. Mag. A. 2003; 83: 2429.

[9] Guvenilir A. and Stock SR. Fatigue \& Fracture of Engineering Materials \& Structures. 1998; $21: 439$.

[10] Yisheng W., Schijve J. Fatigue \& Fracture of Engineering Materials \& Structures. 1995; 18: 917.

[11] e.g. see Donald JK. and Paris PC. Int. J. Fatigue. 1999; 21: 47.

[12] Ludwig W., Buffière J-Y., Savelli S., Cloetens P. Acta mater. 2002; 51: 585.

[13] Ross EL., Kamiski G., Conway JC. Expl. Mech. 1982; 22: 117.

[14] Post D. In: Editor Kobayashi AS. SEAS Handbook on Experimental Mechanics. Chap. 7, Conneticut: Society for Experimental Stress Analysis, 1986.

[15] Lee OS. and Read DT. In: Editors Gomes S. et al. Recent Advances in Experimental Mechanics, Rotterdam: Balkema, 1994; 705.

[16] Cloud G., Paleebut S. Expl. Mech.. 1992; 32: 273.

[17] Machida K. Optical Review. 1997; 4: 253.

[18] Beinert J., Kalthoff JF. In: Editor Sih G. Mechanics of Fracture, 3, London: Noordhoff, 1981; 281.

[19] Pezzotti G., Ichimaru H., Ferroni LP., Hirano K., Sbaizero O. J. American Ceramic Soc.. 2001; 84: 1785.

[20] Shiratori M., Miyoshi T., Nakanishi T., Noda T., Harada M. JSME Int. J. Series I, Solid Mechanics Strength of Materials. 1990; 33: 400.

[21] Kinoshita T. Acta mater. 1998; 46: 3963.

[22] Davidson DL., Hudak SJ. Metall. Mater. Trans. A. 1995; 26: 2247.

[23] Narendran N., Shukla A., Letcher S. Engng. Fract. Mech. 1991; 38: 491.

[24] Smith DG., Smith CW. Engng. Fract. Mech. 1972; 4: 357.

[25] Pindea JT., Krasnowski BR. In: Editor Simpson LA. Fracture Problems and Solutions in the Energy Industry, Springer Verlag, 1982; 147. 
[26] Chiang FP., Asundi A. Optical Engineering. 1982; 21: 570.

[27] Cloetens P., Pateyron-Salome M., Buffière J-Y., Peix G., Baruchel J., Peyrin F., Schlenker MJ. J. Appl. Phys. 1997; 1: 5878.

[28] Buffière J-Y., Maire E., Cloetens P., Lormand G., Fougères R. Acta mater. 1999; 47: 1613.

[29] ASTM E647, Annual Book of ASTM Standards, Section 3, Vol.03.01. Pennsilvania: ASTM, 1995; 578.

[30] Herman GT. In: Image Reconstruction from Projections, The Fundamentals of Computerized Tomography, Orland: Academic Press, 1980.

[31] Labbe C., Froment JC., Kennedy A., Ashburner J., Cinotti L. Alzheimer Disease \& Associated Disorders. 1996; 10: 141.

[32] Kim Y., Zhu XK., Chao YJ. Engng. Fract. Mech.. 2001; 68: 895.

[33] Andersen TL. In: Fracture Mechanics. Boca Raton: CRC Press, 1995.

[34] Xiao QZ., Karihaloo BL. Engng. Fract. Mech. 2001; 69: 959.

[35] Hartranft RJ., Sih GC. J. Math. Mech.. 1969; 19: 123.

[36] Reed PAS., Sinclair I., Loo M., Wu XD. In: Final Report on MoD Research Contract Ref. No.2040/515/ MA, Research Report - PR/RA/94/08/171, Southampton: University of Southampton, 1994.

[37] Roth SD. Computer Graphics and Image Processing. 1982; 18: 109.

[38] Jani AB., Pelizzari CA., Chen, GTY., Grzeszczuk RP. J. Computer Assisted Tomography. 1998; 22: 459.

[39] Trockels I., Gysler A., Lutjering G. In: The fourth Int. Conf. On Aluminium Alloys, 1994; 717.

[40] Gran FJ., Gysler A., Lutjering G. In: Editors Bailon JP., Dickson JI. Fatigue '93, 1993; 623.

[41] Sassen K. Bulletin of the American Meteorological Society. 1991; 72: 1848.

[42] Zhai T., Wilkinson AJ. and Martin JW. Acta mater. 2000; 48 : 4917. 


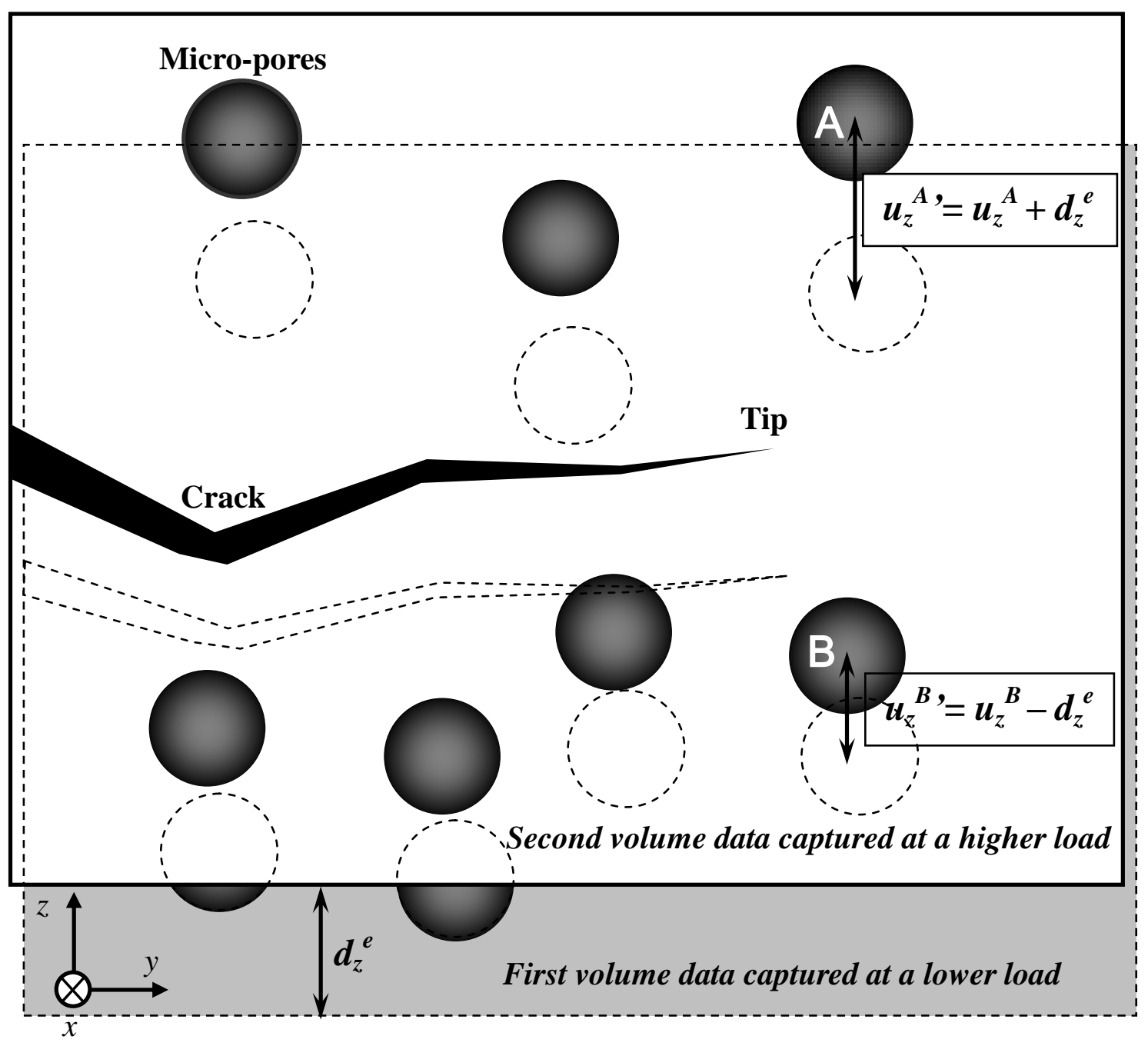

Fig.1 Schematic illustration of the bipolar separation of the mode I local stress intensity factor measured at each micro-pore. Incomplete registration between a couple of volume data, $d_{z}^{e}$, is added on the true displacement of micro-pore $\mathrm{A}, u_{z}^{A}$, in the upper quadrant, while it is subtracted from that of micro-pore $\mathrm{B}, u_{z}{ }^{B}$, located in the lower quadrant. 


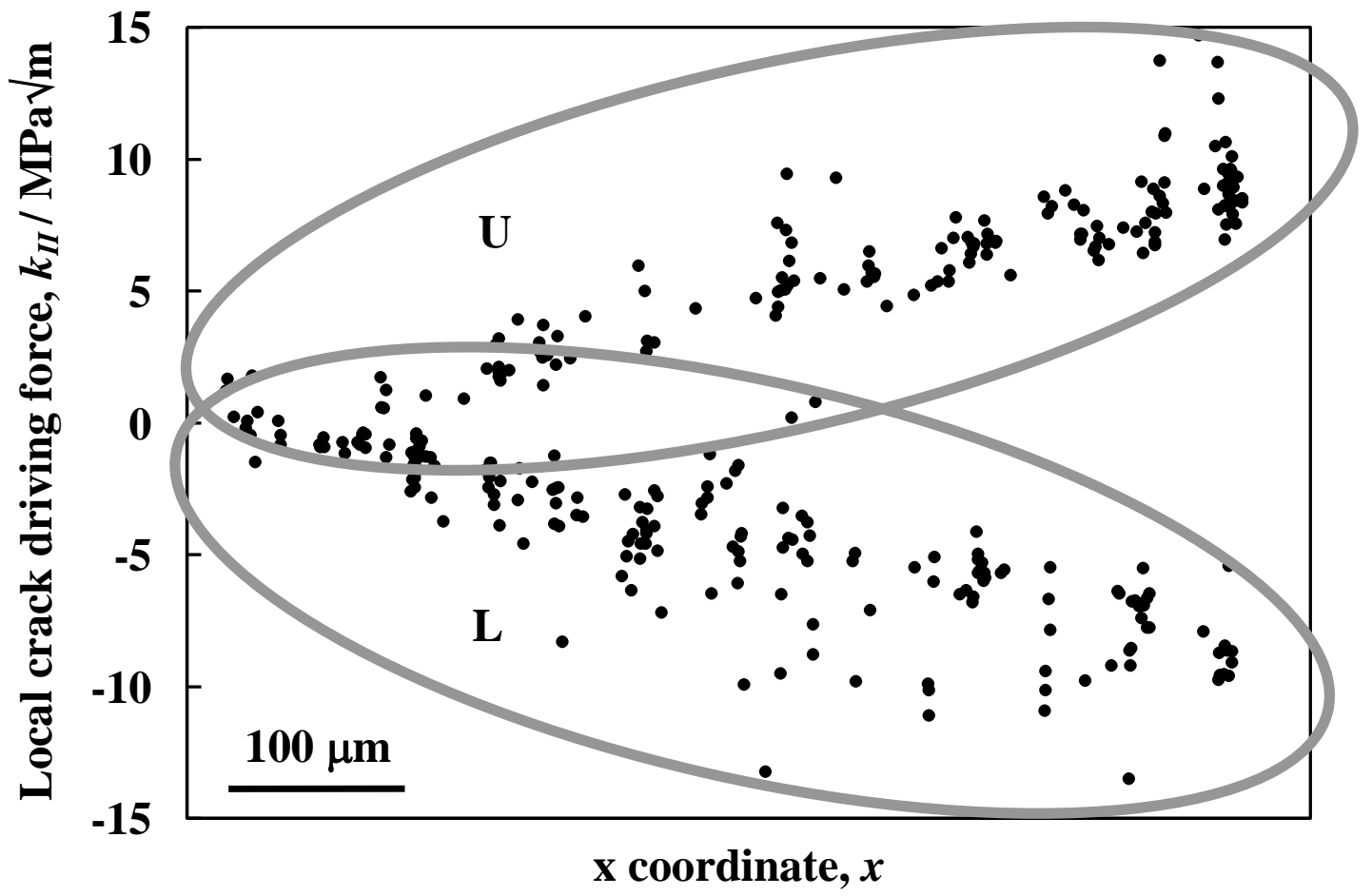

Fig.2 Variation of mode II local crack driving force, $k_{I I}$, along crack front line at applied $K_{I}$ of $4.69 \mathrm{MPa} \sqrt{\mathrm{m}}$. The data were plotted without applying calibration coefficients for registrating the couple of volume data captured at the two different load levels for demonstration purpose. Regions $\mathrm{U}$ and $\mathrm{L}$ are the groups of data points which were calculated by micro-pores in the upper and lower quadrants in Fig.1, respectively. 


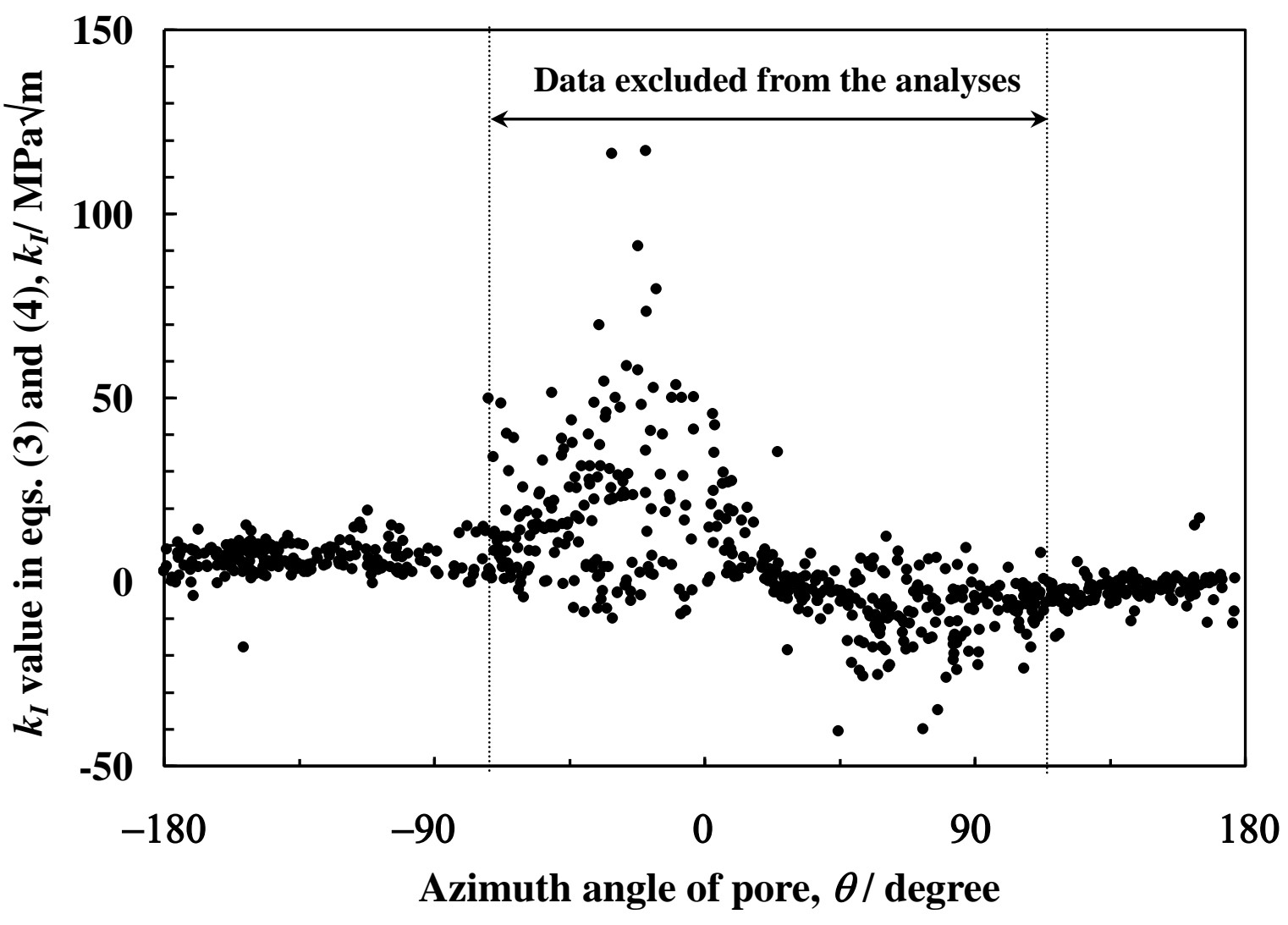

Fig. 3 The effects of azimuth angle of micro-pore on $k_{I}$ in the right-hand side of equations (3) and (4), representing the existence of non-linear deformation ahead of a crack-tip (i.e. $-71 \sim+111$ degrees). The data taken at applied $K_{I}$ of $4.69 \mathrm{MPa} \sqrt{\mathrm{m}}$ were used for the calculation. 


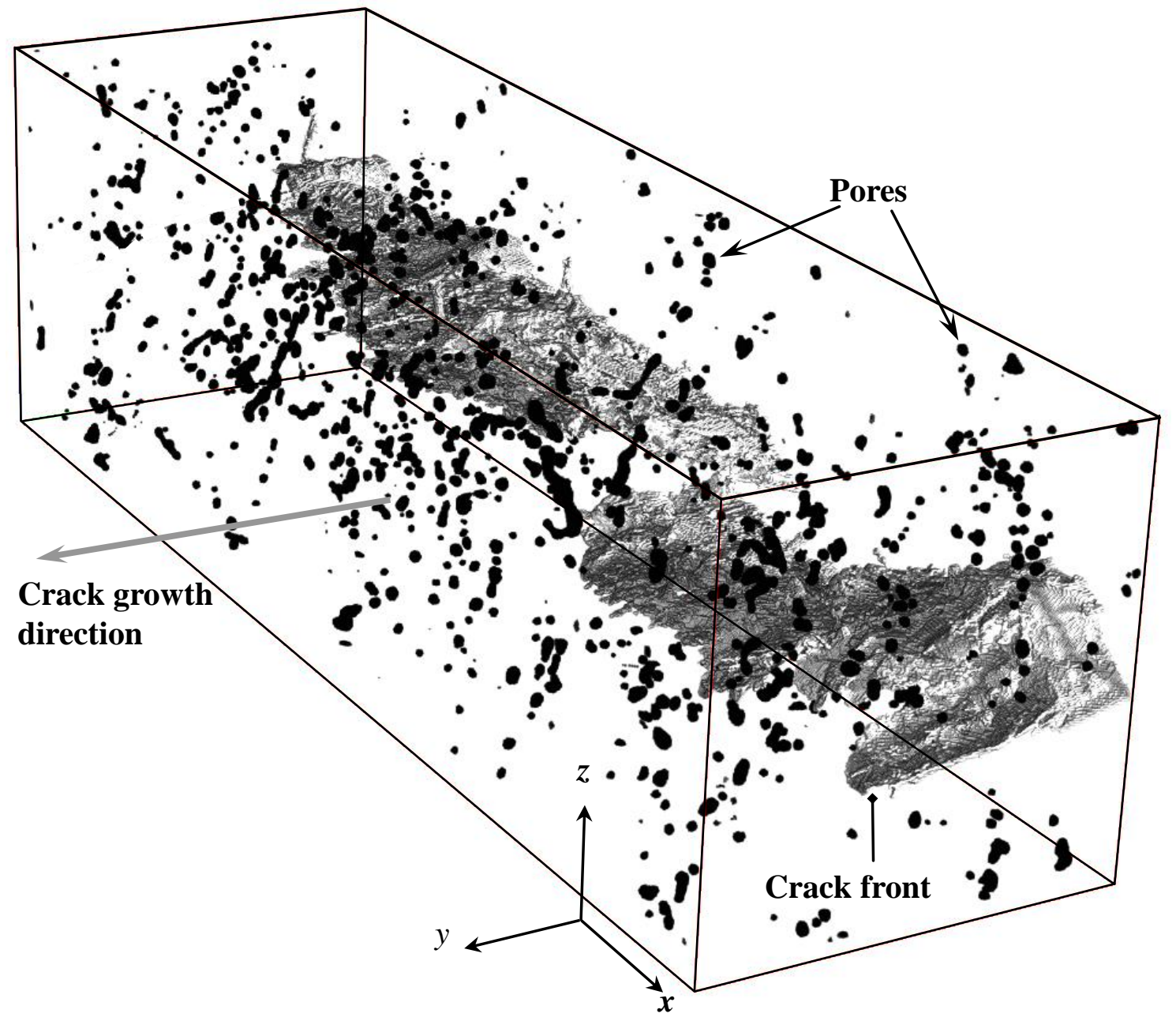

Fig.4 3D perspective view of a crack and pore distribution in the analysed sample volume which were rendered by ray tracing algorithm $(910 \times 270 \times 270$ voxels), representing a crack-tip and its vicinity after aluminium has been removed from the volume. 


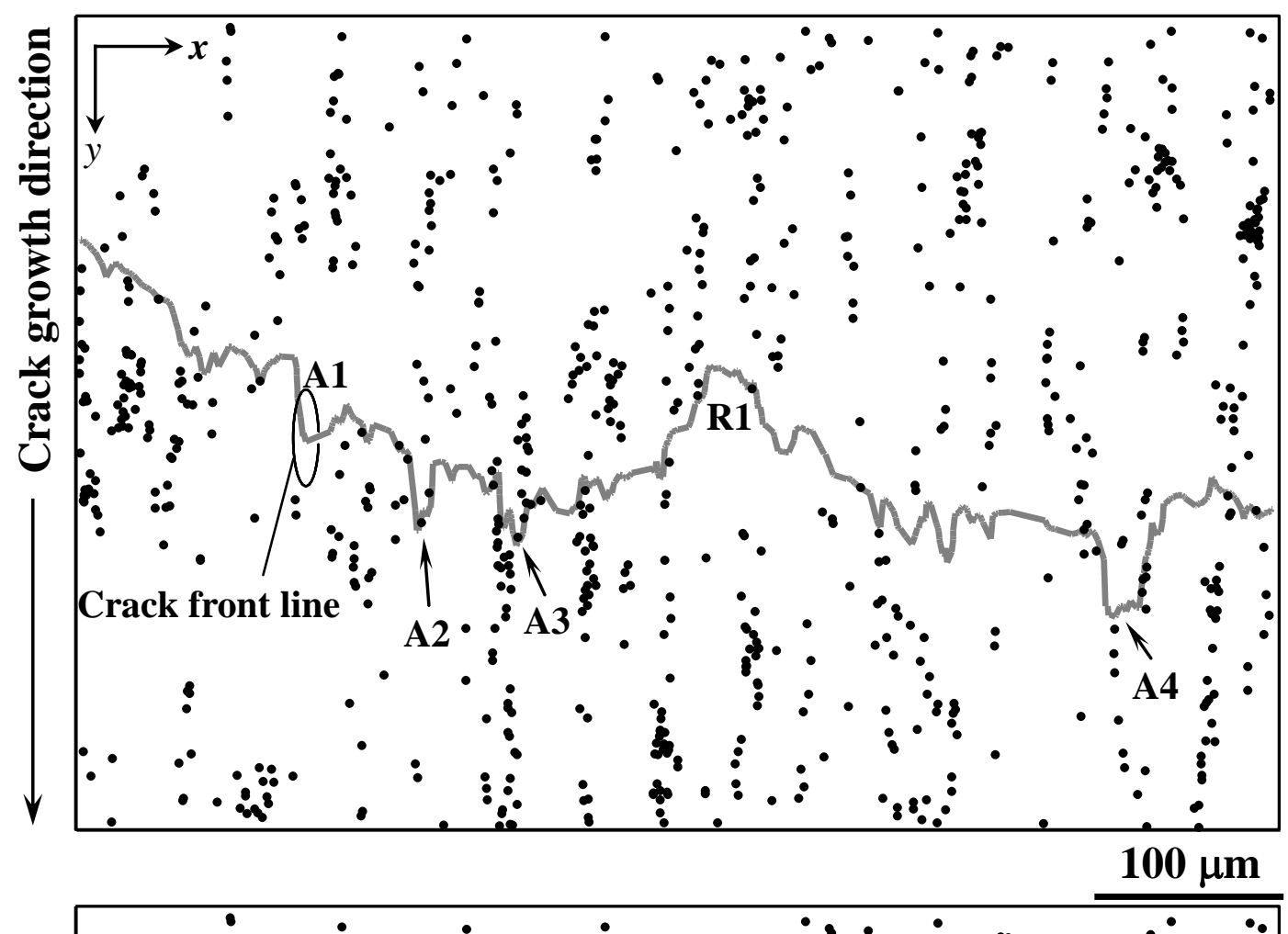

Fig. 5 Two-dimensional projection of all the micro-pores shown in Fig. 4 onto a plane perpendicular to the loading axis. The crack front line was also superimposed in grey by projecting the crack front line onto the same plane as the micro-pores.

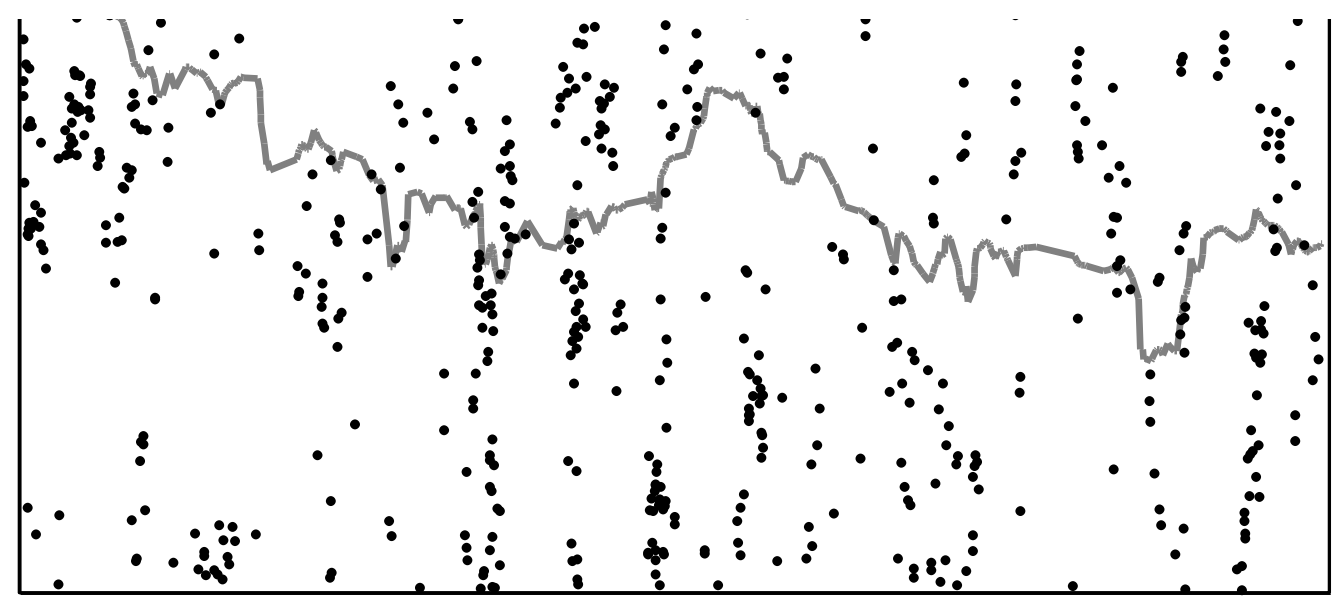


(a)

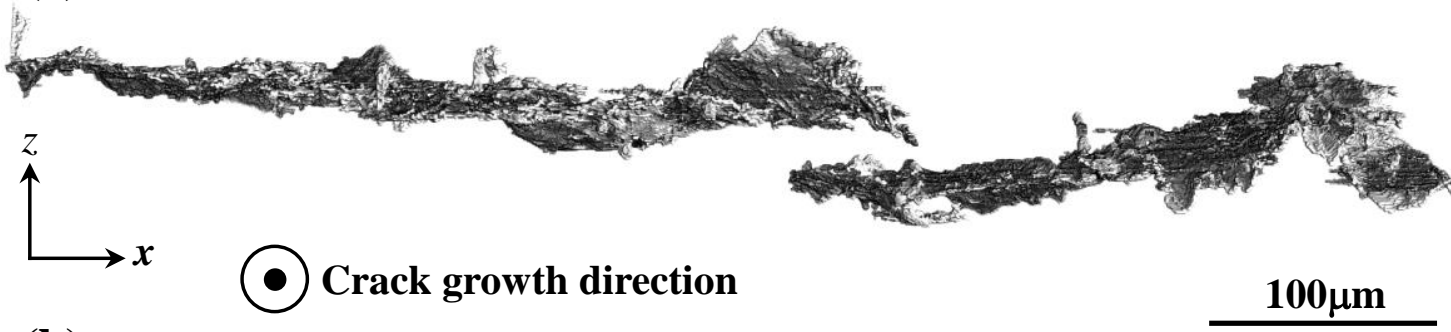

(b)

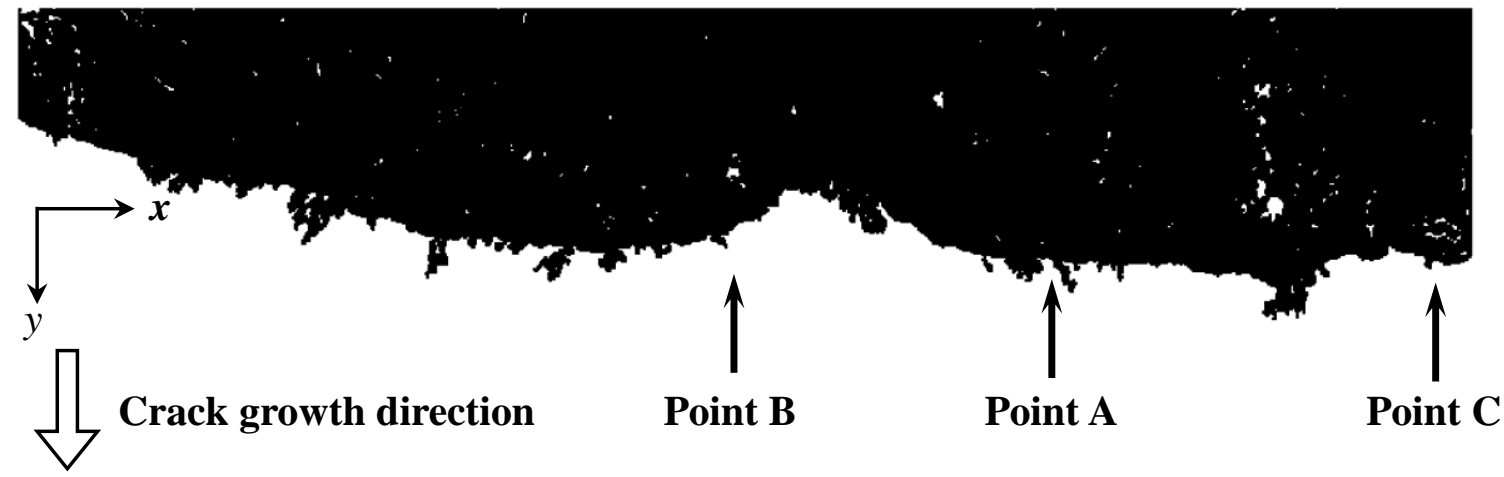

Fig. $63 \mathrm{D}$ perspective views of the crack taken at applied $K_{I}$ of 0.42 $\mathrm{MPa} \sqrt{\mathrm{m}}$. (a) is a ray tracing representation viewed in the $y$ direction which emphasizes surface topology, and (b) is a ray casting representation viewed in the $z$ direction in which crack face contact is highlighted in white. 

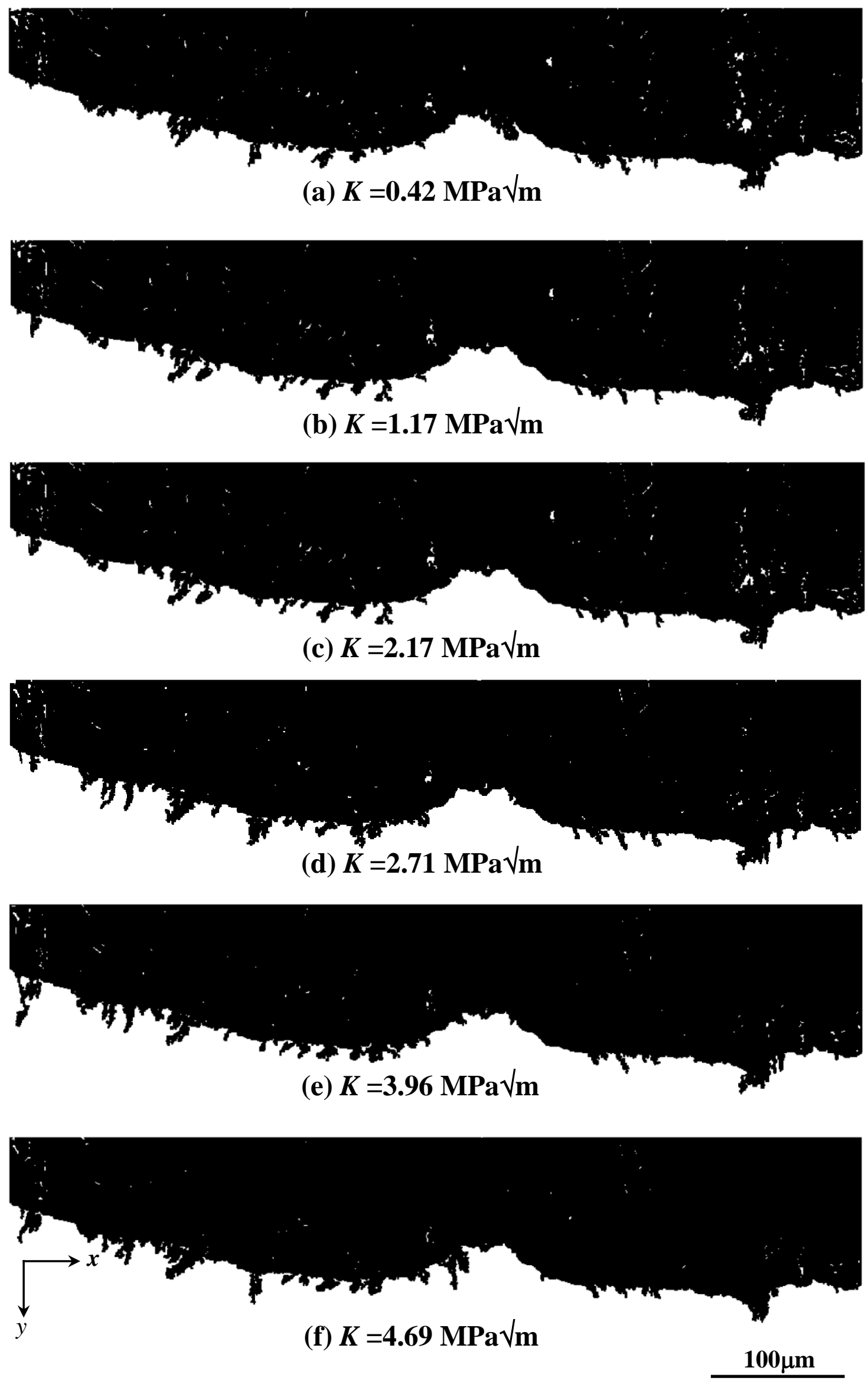

Fig. 7 Change in the spatial distribution of crack surface contact viewed in the $z$ direction, where the areas of contact are highlighted in white. 


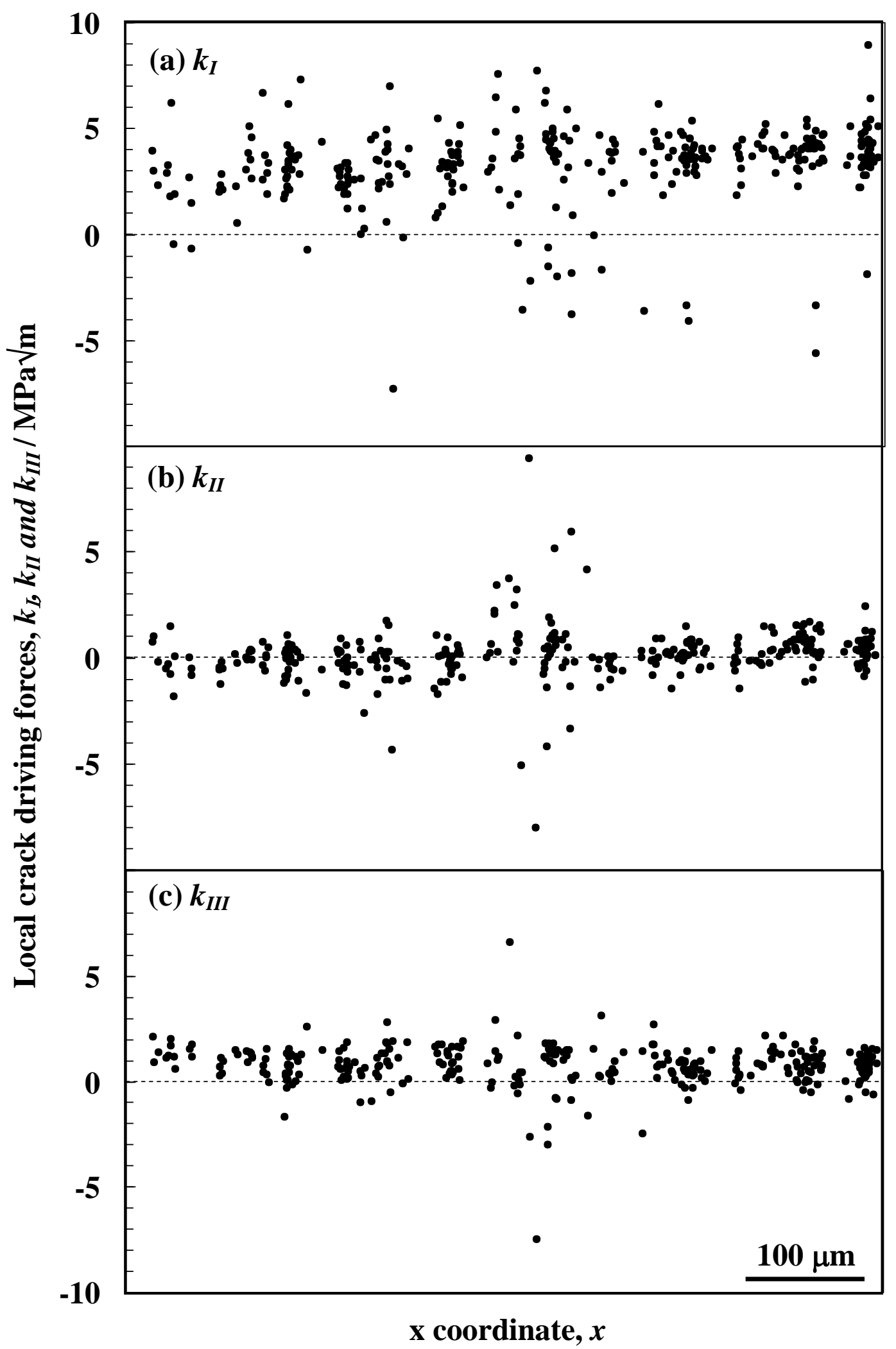

Fig. 8 Variations of modes I, II and III local crack driving forces along the crack front line which were measured at applied $K_{I}$ of $4.69 \mathrm{MPa} \sqrt{\mathrm{m}}$. The data were plotted after applying the calibration functions. 


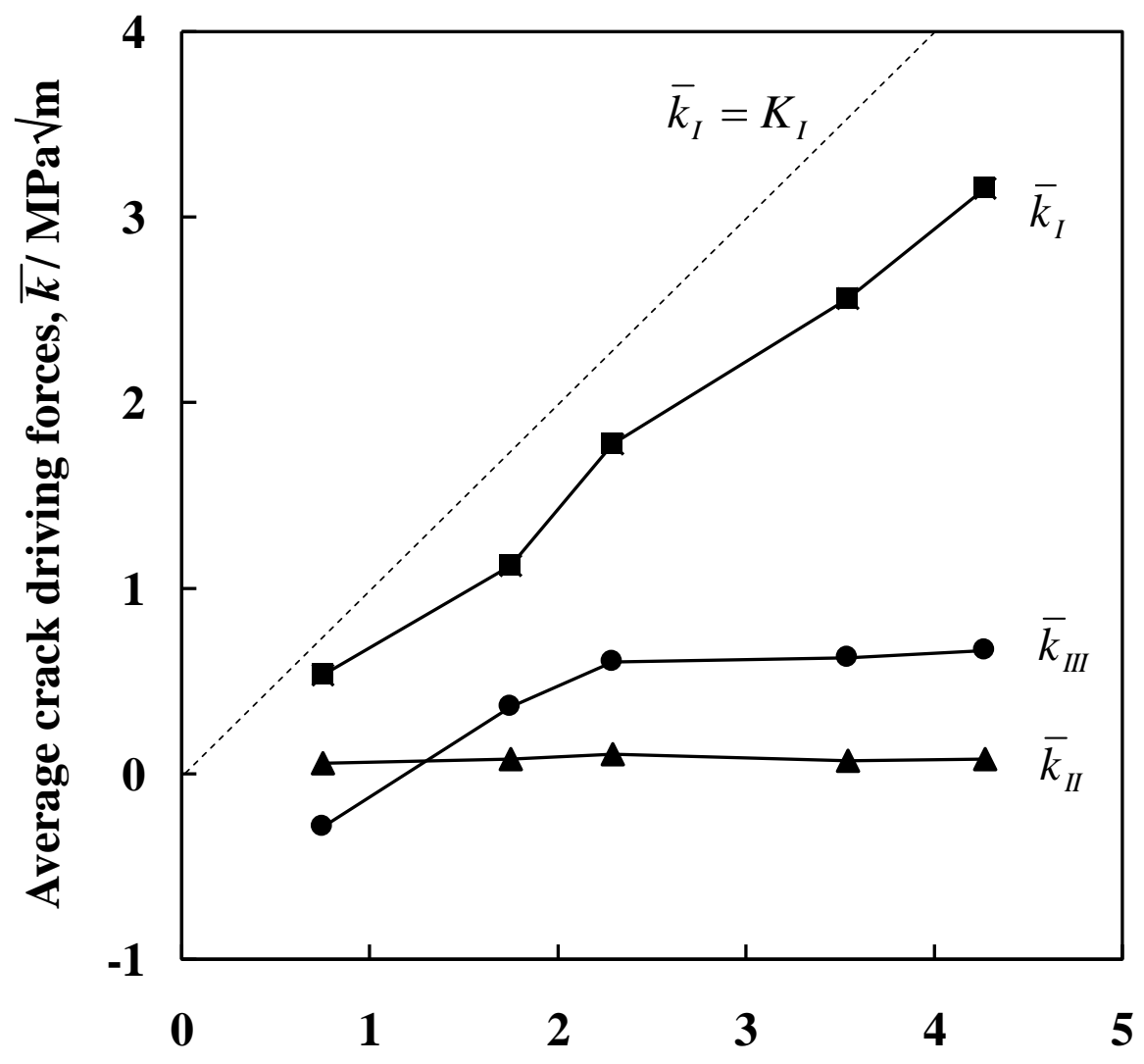

Applied stress intensity range, $K-K_{\min } / \mathrm{MPa} \sqrt{\mathrm{m}}_{\mathrm{m}}$

Fig. 9 Variations of modes I, II and III local crack driving forces as a function of applied stress intensity range. The data were averaged along the whole crack front line. 


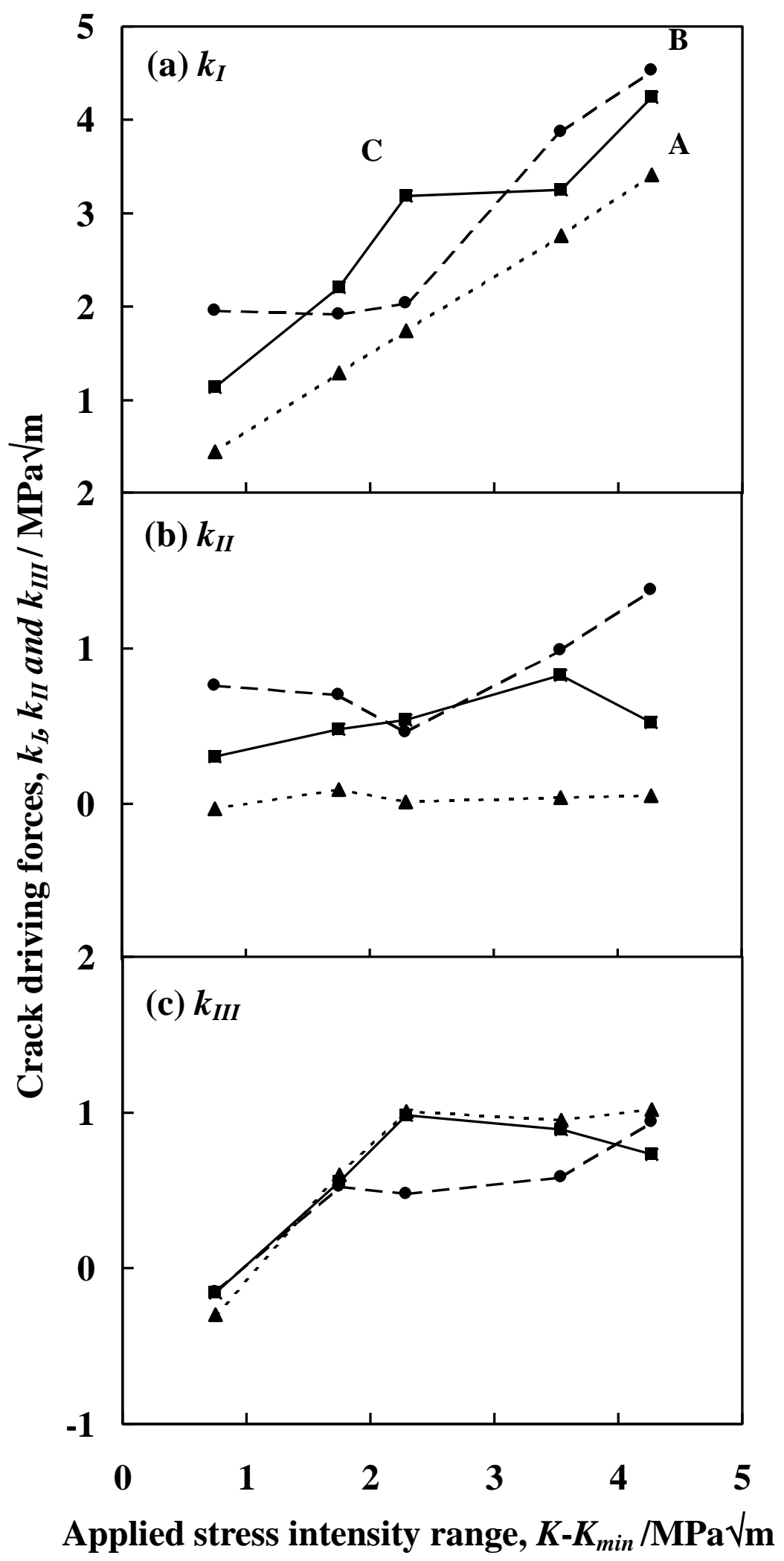

Fig. 10 Variations of modes I, II and III local crack driving forces at points $\mathrm{A}, \mathrm{B}$ and $\mathrm{C}$ as a function of applied stress intensity. Locations of the points are shown in Fig. 6. 


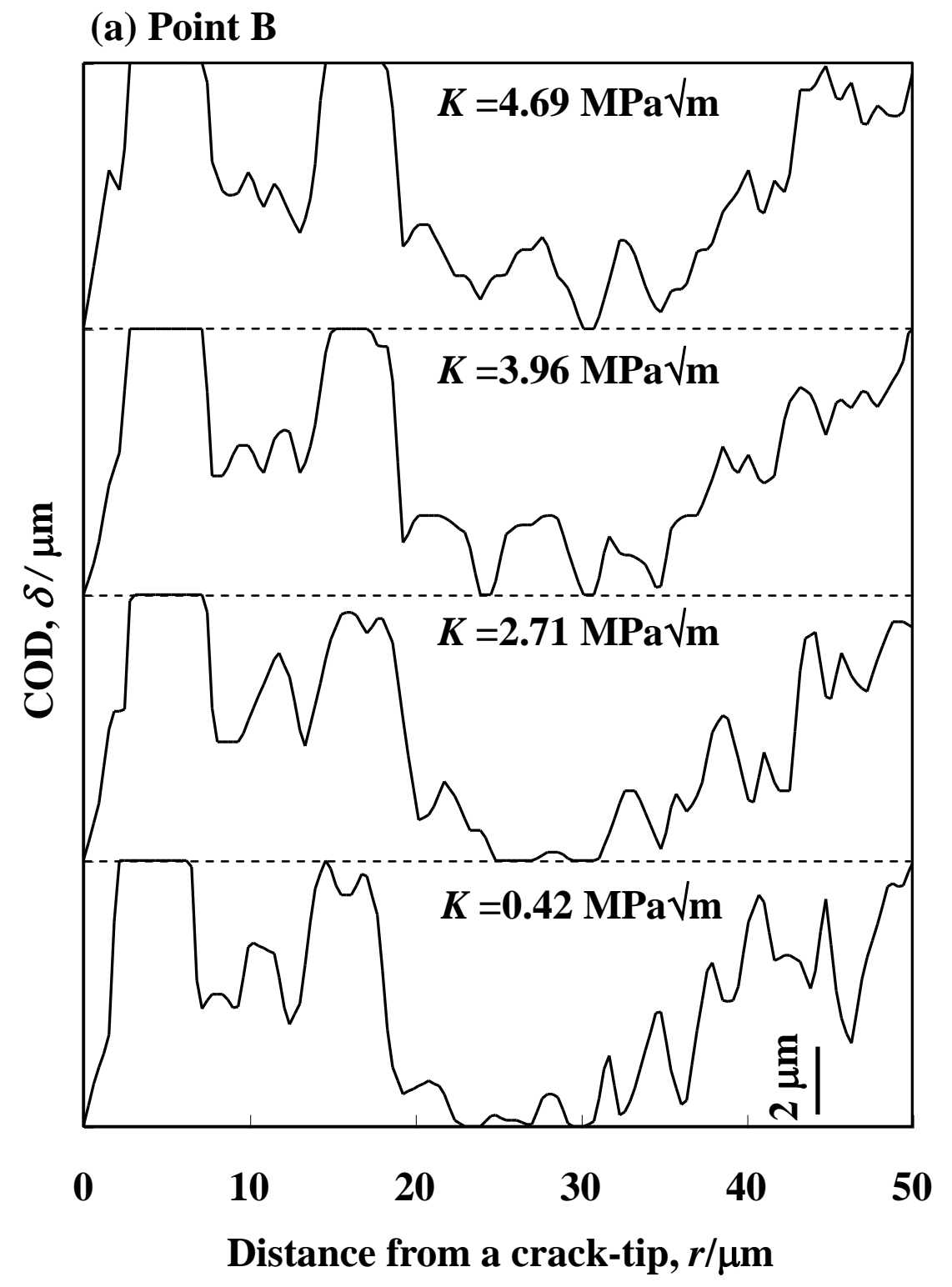


(b) Point C

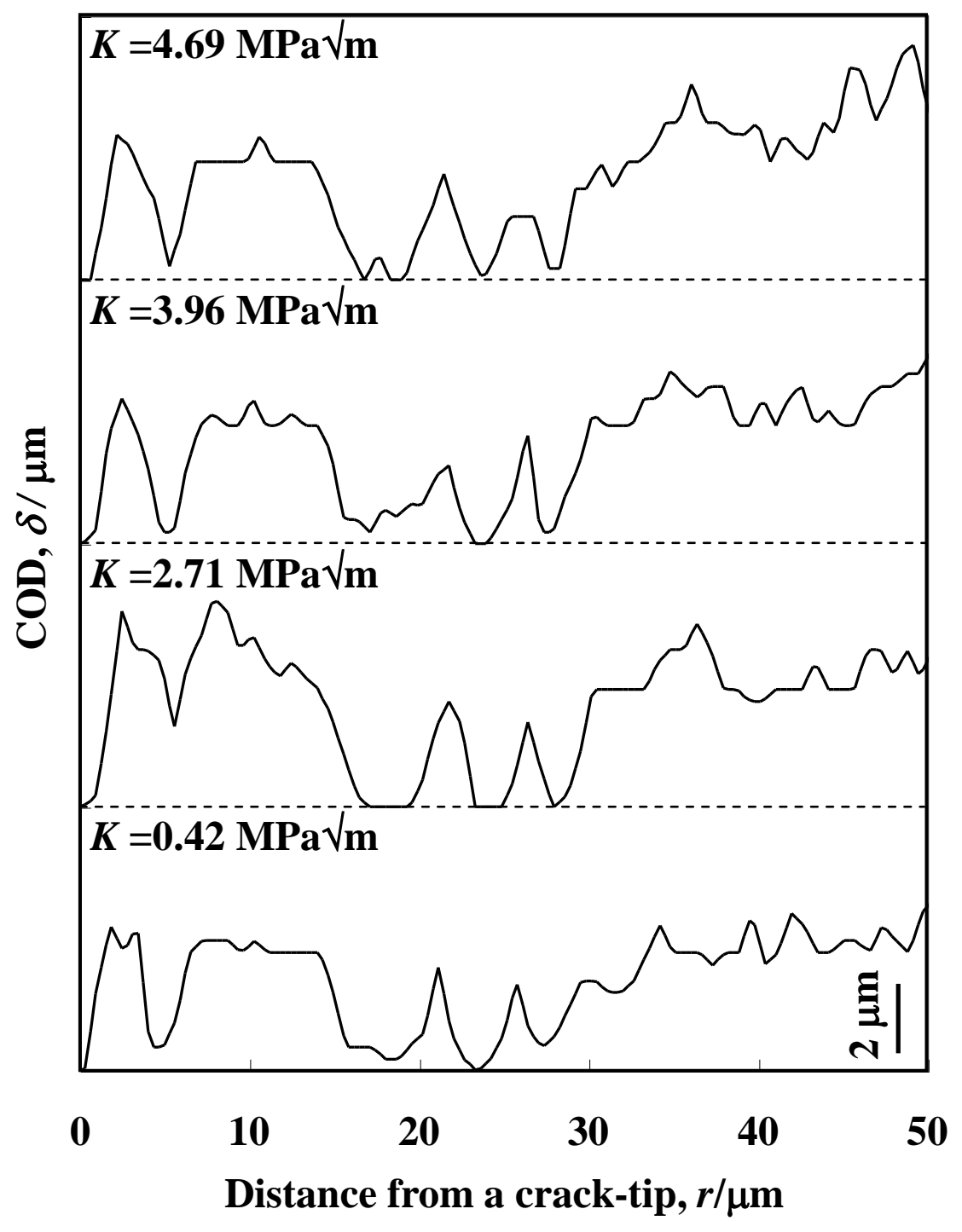

Fig. 11 The variations of crack opening profile on the cross-sections corresponding to (a) point B and (b) point $\mathrm{C}$ in Fig.6. The profiles were quantified utilising the ray casting algorithm ploting back from the crack-tip, 